\title{
Generalization of Uniform Continuity in Functional Analysis
}

\author{
Jin Chen* \\ Academic Affairs Office, Zhaotong University, Yunnan, 657000, China \\ *Corresponding Author email: 84754919@qq.com
}

Keywords: Uniformly continuous; identical continuous; sequentially convergent

\begin{abstract}
When studying a continuous model theorem in functional analysis, we come across an indirect proof method. For example, the proof of compactness theorem is extremely abstract and complicated. An indirect proof method is given for a continuous mode theorem in nonlinear functional analysis. Weakly consistent continuous and consistently continuous definitions make it easier for you to compare and better analyze consistent and continuous definitions. In the functional analysis, there are metric spaces, linear normed spaces and inner product spaces. The following is a brief description of the consistent continuity in the metric space. In the functional analysis, there are also concepts such as strong convergence, weak convergence, and weak convergence of functional sequences. On the basis of topological sequence, this paper solves the critical path by calculating the earliest occurrence time of each vertex and marking the corresponding path. For example, the proof of compactness theorem is extremely abstract and complex. Because functional analysis is a generalization of mathematical analysis, the definition of uniform continuity in mathematical analysis is extended to linear normed spaces.
\end{abstract}

\section{Introduction}

In the early 1960s, Rob1snon created nonstandard analysis, which is a nonstandard model in mathematical logic. The proof of basic theorems, such as the compactness theorem, is extremely abstract and complex [1].

When we study a continuous model theorem in functional analysis, we are exposed to an indirect proof method [2].Let $\mathrm{f}(x)$ be a function defined in interval I. If $D_{L}>0$, there is $1(9)>0$, let $D$ and $L$ be real normed linear spaces. $d$ is the operator from $D$ to $L$, and uses $D_{L}\left(x_{L}\right)$ to represent the domain of $D_{R}$, which is written as: $D_{L} \quad D \rightarrow L$, obviously $L$ is a monotonous positive function, and

$$
\left|D_{L}\left(x_{L}\right)-D_{R}\left(x_{L}-D_{L}\left(x_{L}\right)\right)\right|<1(9)
$$

If $w$ is uniformly equal on exp, then $d_{g}$ divided by $r_{g}$ is meaningful in terms of two additions [3-5]. Since $\mathrm{w}$ is uniformly continuous on $x$, there are: For definition $y>0$, there is $d>0$. Then there are: $d_{g}, r_{g}, t(x, y \in d)$ First take the positive integer exp, so that $(x, y, d)$, thus satisfying any of the following equations, then:

$$
w(x, y, d)=\exp \left(-\left(\frac{d_{g}}{r_{g}}+\frac{d_{c}}{r_{c}}\right)\right)
$$

Let $\left(D_{L}\left(x_{L}\right)\right.$ be a mapping on the non-empty metric space $x_{L} D_{L} x$ to the real field R (called a functional or function), $D \quad \mathrm{X}$, if there is an arbitrary $\mathrm{x}_{\mathrm{L}}>0$, there is always a $D_{R}>0$. So that any $D_{L}$, $D_{R}, D$, as long as $D\left(\mathrm{x}_{\mathrm{L}}\right)<1$, can be expressed by the following inequalities:

$$
\left|D_{L}\left(x_{L}\right)-D_{R}\left(x_{L}-D_{L}\left(x_{L}\right)\right)\right|<1
$$

$w(t)$ is defined and consistent on $w_{2}$, and $w 1$ is obtained. For any $t, w 1$ is defined, $w(t)$ is t. Then $w 2$ is not equal to $\mathrm{w}(\mathrm{t})$, which can be derived from the following formula: 


$$
w(t)=w_{2}+\left(w_{1}-w_{2}\right) \frac{T-t}{T}
$$

\section{Proof result}

Because dmax is uniformly continuous on $\mathrm{i}, \mathrm{i}>0,1 . . \mathrm{n}$ max and $\mathrm{i}=1 . . \mathrm{N}$ has $\mathrm{dmax}<\mathrm{di}$. So $1 . . \mathrm{n}>0$, $\operatorname{dmax}>0$, $d i \in 1$..n and $\operatorname{dmax}<\mathrm{i}$, there is $\operatorname{dmax} \leq \max , \mathrm{i}=1$.nn, that is, dmax is also consistent. Look at the following formula:

$$
d_{\max }=\max \left\{d_{i}\right\}, i=1 . . n
$$

Since $x i(t)$ is uniformly continuous on $X, n i>0, x>0$, let $x(t), n i(t) \in x i$, and $x i-x(t)<0$, $n i \in 1, x i(t)(x i) . X(t)+n i(t)<1$, so fixed $x i$, that is, $x i(t)$ uniformly continuous functional on $X$, can be obtained by the following formula:

$$
x_{i}(t)=x(t)+n_{i}(t)
$$

a is a linear bin space b, then set to (ATA), ATB is a, which defines [a,b,c]T as AT, then A is equal to a, denoted as ATA=ATB. This equality relationship is The equivalence relationship, which attributes all elements equal to AT to an equivalent class. The ATB is denoted by the following expression if the set of all the elements (i.e. equivalent classes) denoted by (ATB) $=[a, b, c]$ is denoted as AT:

$$
[a, b, c]^{T}=\left(A^{T} A\right)^{+} A^{T} B
$$

Definition: Let any $d(1 y)$ be $[x, y]$. Where ax is by. The norm in c. Obviously $(x, y)$ satisfies the following three conditions: for any $d(2 j)(x, y)$ for any $d(i, j),[x, y]$ and for any $d(n, j)$. Therefore, we call ay + by $+c$ a linear over-expansion space where $d(n, j)(x, y) d(i, j)$ is ax. For any by $c$, the constant column $d(1, j)(x, y)$ is also a unit of by, we consider $c$ as $d(2, j)$.Therefore, space $c$ can be regarded as the subspace of space by, that is, the expansion of $a x+b y+c$ is $(x, y) d(n, y)$ is finite, with $(x, y)$ (infinitesimal). The following expressions can be expressed as follows:

$$
\begin{gathered}
d^{(1, j)}(x, y)=a x+b y+c \\
d^{(2, j)}(x, y)=a x+b y+c \\
\vdots \\
d^{(i, j)}(x, y)=a x+b y+c \\
\vdots \\
d^{(n, j)}(x, y)=a x+b y+c
\end{gathered}
$$

\section{Uniform continuity in metric spaces}

In the functional analysis, there are metric spaces, linear normed spaces and inner product spaces. The following is a brief description of the consistent continuity in the metric space. If $A$ is a continuous mapping from the compact subset $\mathrm{Z}$ of the metric space $\mathrm{P}$ to the metric space $\mathrm{S}$, it is proved that a must be uniformly continuous mapping.

$$
\alpha_{i j}=P\left(Z_{t+1}=S_{j} \mid Z_{t}=S_{i}\right)
$$

In functional analysis, whether a mapping can extend to the whole space is a very complicated problem. Uniformly continuous mappings on bounded closed sets are not uniformly continuous. The image of the oscillating function is shown in Figure 1: 


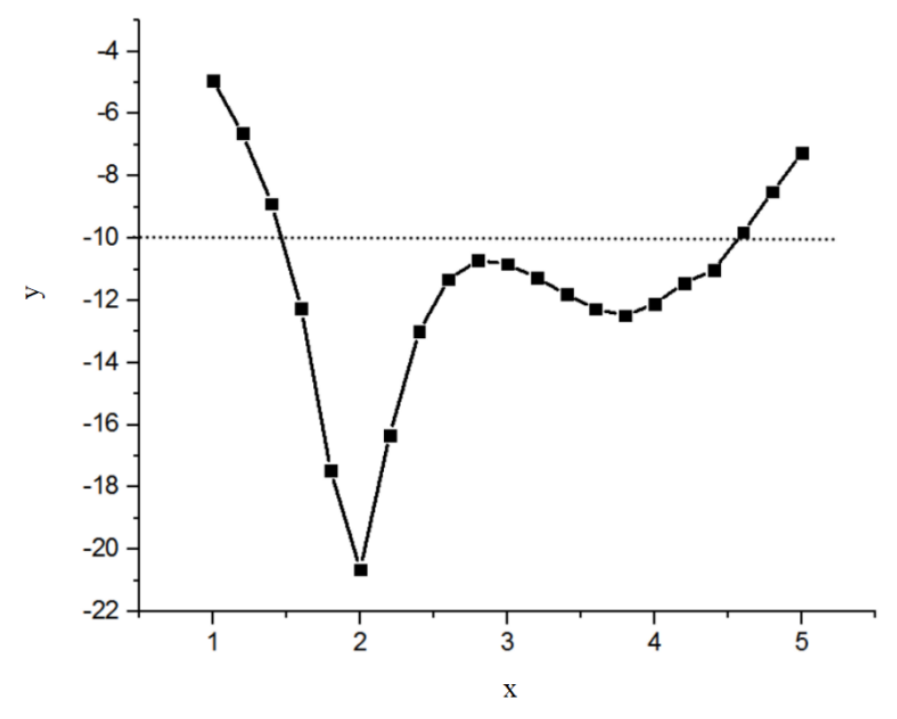

Figure 1 Image of the oscillation function

The extension considered below is to extend the consistent continuous mapping defined on A to B. This extension principle is obvious from the perspective of scalability, but it has both theory and application. A certain meaning.

The maximum absolute error of the compact difference scheme is shown in Table 1.

Table 1 Tight feature difference format maximum absolute error

\begin{tabular}{cccc}
\hline & $\mathrm{t}=0.5$ & $\mathrm{t}=0.3$ & $\mathrm{t}=2$ \\
\hline $\mathrm{Re}=5$ & $4.3 \times 5^{-5}$ & $2.9 \times 5^{-5}$ & $3.2 \times 5^{-5}$ \\
$\mathrm{Re}=10$ & $4 \times 5^{-5}$ & $2.6 \times 5^{-4}$ & $9.8 \times 5^{-4}$ \\
$\mathrm{Re}=20$ & $3.8 \times 5^{-5}$ & $5.7 \times 5^{-3}$ & $1.5 \times 5^{-3}$ \\
\hline
\end{tabular}

Let $r$ be a Banach space, qi be the finite-dimensional subspace of $i, P$ be the pre-space of qi, and $s$ be a consistent continuous map defined on $\mathrm{P}$. Then there is a unique continuous mapping y on qi, such that:

$$
r_{t}(i)=P\left(q_{t}=s_{i} \mid y_{t}\right)
$$

This definition of $\mathrm{P}$ has nothing to do with the selection of basic columns converging to $\mathrm{X}$. If $\mathrm{R}$ exists, the $\mathrm{P}$ port converges to another basic line of $\mathrm{X}$ :

$$
P(X \leq R)=P
$$

Let $\mathrm{x} 1$ be the linear operator on $\mathrm{w}$, then the continuous and necessary condition of 1 is: for any $\mathrm{z} 1=\mathrm{vi} . \mathrm{x} 1+\mathrm{ti}$. Proof (necessity) Let 1 be continuous, then $(\mathrm{i}, \mathrm{x}, \mathrm{y})$ is bounded, that is, there is $\mathrm{xi}$, so that any $\mathrm{x}=(\mathrm{i}, \mathrm{x}, \mathrm{y})$ then $\mathrm{zl}=\mathrm{zl} . \mathrm{x} 1+\mathrm{t} 1$ : represented by the following formula:

$$
\begin{gathered}
x_{l}=\left\langle w, g_{l}\right\rangle \\
l=(i, x, y) \\
z_{l}=v_{i} \cdot x_{l}+t_{i}
\end{gathered}
$$

The comparison diagram between approximate solution and exact solution is shown in Figure 2 


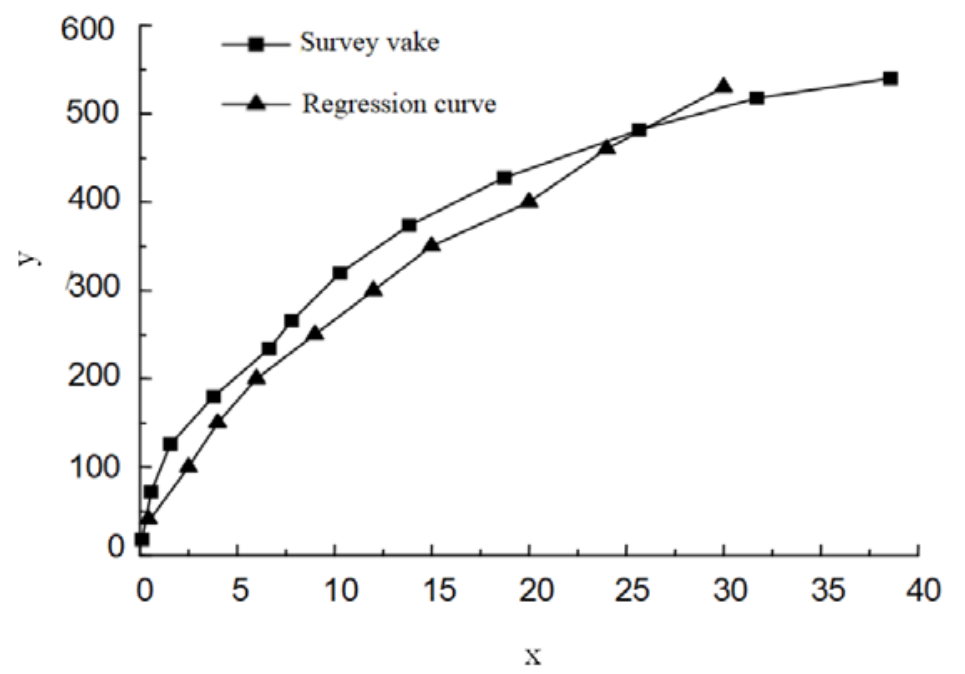

Fig. 2 Comparison of Approximate Solutions and Precise Valves for Conformal Features

Obviously $\mathrm{M}$ is an infinite subset of $\mathrm{R} 0$, which is represented by the following equation:

$$
M\left(t_{0}\right)=R_{0}\left(1+\frac{2 \Delta R U}{Q_{0} R_{0}^{2}} t_{0}\right)^{0.5}
$$

The multi-step feature scheme can achieve higher accuracy than the single-step feature difference scheme and get better results.

Table 2 Maximum absolute error calculated from multi-step feature difference format

\begin{tabular}{ccc}
\hline $\mathrm{t}$ & $\mathrm{h}$ & $\mathrm{c}$ \\
\hline $5^{-3}$ & $10^{-4}$ & $3.2701 \times 10^{-3}$ \\
$5^{-3}$ & $10^{-4}$ & $2.0246 \times 10^{-4}$ \\
$5^{-3}$ & $10^{-5}$ & $2.0185 \times 10^{-6}$ \\
$5^{-3}$ & $10^{-5}$ & $2.0257 \times 10^{-8}$ \\
\hline
\end{tabular}

\section{Conclusion}

So far, we have proved the inequalities of the matrix ranks common to the claws by the method mentioned at the beginning. For the problem of consistent continuity of functions, we propose and prove the consistent discriminant method and ratio discriminant method of the decision function, which greatly simplifies and broadens the discriminable range of the consistent continuity of the function. In this paper, on the basis of function comparison discriminant method, the application of comparison discriminant method is extended in n-dimensional Euclid space, thus solving the problem of judging the uniform continuity of multivariate functions. The conclusion of this paper enriches the consistent and continuous theoretical results of multivariate functions. From the proof of the specific problem, we can also see that another advantage of this method is that it does not need to give inequalities to prove. Due to the flexible "combination" of matrix block multiplication, we can construct this method by using this method. Many inequalities of matrix ranks, and give the condition that the equal sign holds.

\section{References}

[1] Niwa Y, Tomita H, Satoh M, et al. A Three-Dimensional Icosahedral Grid Advection Scheme Preserving Monotonicity and Consistency with Continuity for Atmospheric Tracer Transport[J]. Journal of the Meteorological Society of Japan, 2011, 89(3):255-268.

[2] Kalisch B J, Begeny S, Anderson C. The effect of consistent nursing shifts on teamwork and continuity of care[J]. Journal of Nursing Administration, 2008, 38(3):132-137.

[3] Langov A, Mollov S. Positive psychosis symptom structure in the general population: Assessing 
dimensional consistency and continuity from 'pathology' to 'normality'[J]. Psychosis, 2010, 2(3):199-209.

[4] Dixon P. British policy towards Northern Ireland 1969-2000: continuity, tactical adjustment and consistent 'inconsistencies' $[\mathrm{J}]$. British Journal of Politics \& International Relations, 2001, 3(3):340-368.

[5] Lortie-Lussier M, Côté L, Vachon J. The Consistency and Continuity Hypotheses Revisited through the Dreams of Women at Two Periods of their Lives[J]. Dreaming, 2000, 10(2):67-76. 